\title{
LES ACQUIS DE LA FORMATION DOCTORALE : PERCEPTIONS DES COMPÉTENCES DÉVELOPPÉES
}

\author{
MARCELLINE BANGALI \\ UNIVERSITÉ LAVAL
}

Remarque : une traduction anglaise apparaît après la liste des références.

\section{Résumé}

Plusieurs travaux soulignent des difficultés particulières auxquelles certains titulaires d'un doctorat sont confrontés sur le marché du travail en dehors du milieu universitaire. Une des principales raisons de ces difficultés serait la méconnaissance ou l'inadéquation des acquis de la formation doctorale en ce qui concerne les compétences recherchées par les organisations. Or, en dehors de données statistiques, peu de travaux nous renseignent sur les perceptions que les différents acteurs ont de ces compétences. Cet article apporte une contribution dans ce sens. Il est basé sur les résultats d'une recherche mixte à devis séquentiel. La première étape a consisté en une étude qualitative par entretiens semi-directifs réalisés auprès de 85 diplômés du doctorat en emploi et 21 responsables d'organisations. Les résultats de cette étude, dont les données ont été traitées par la méthode Alceste, ont servi à la conception d'une échelle de 45 items sur les compétences des titulaires d'un doctorat. Cette échelle a été mesurée lors de deux enquêtes par questionnaire auxquelles ont répondu 2139 diplômés du doctorat en emploi et 215 responsables d'organisations. Des analyses descriptives de comparaison de moyennes standardisées ( $d$ de Cohen) mettent en évidence des points de convergence qui montrent que la formation doctorale pourrait constituer un atout pour le développement des compétences du futur, notamment celles difficiles à automatiser : la gestion de la complexité, la créativité, l'esprit critique.

Mots-clés : doctorat, transition, compétences, compétences du futur, intentionnalité, employabilité

\section{Abstract}

A number of studies point to particular challenges that some $\mathrm{PhD}$ graduates face in the labour market outside of academia. One of the main reasons for these difficulties is said to be a lack of knowledge or inadequacy of what doctoral graduates have acquired in terms of the skills sought by employers. However, apart from statistical data, there is little work that tells us about the perceptions that the various groups and individuals involved have of these skills. This article makes a contribution in this direction. It is based on the results of a sequential mixed methods study. The first stage consisted of a qualitative study using semi-structured interviews of 85 employed PhD graduates and 21 organizational leaders. The results of this study, whose data were processed using the Alceste method, were used to design a 45-item scale on the skills of doctoral graduates. This scale was measured in two questionnaire surveys completed by 2,139 employed doctoral graduates and 215 organizational leaders. Descriptive analyses comparing standardized averages (Cohen's $d$ ) highlight points of convergence that show that doctoral training could be an asset for the development of future skills, especially those that are difficult to automate: complexity management, creativity, critical thinking.

Keywords: PhD, transition, skills, future skills, intentionality, employability

\section{Introduction}

Les réflexions développées dans cet article sont en résonance avec des témoignages relayés par les médias sur les difficultés d'insertion professionnelle des titulaires d'un doctorat (Braün, 2014; Charbonneau, 2019; Charrette, 2014; Dalmont, 2018; Tamburri, 2013). Au Canada, les problématiques liées à l'employabilité de ces diplômés préoccupent de plus en plus (Didiano et al., 2019; Edge et Munro, 2015; McAlpine et al., 2018; Por- 
ter et al., 2017; Reithmeier et al., 2019). Selon certains auteurs (Maldonado et Wiggers, 2013; Mendoza, 2007), ces difficultés seraient en partie liées au contexte de la formation doctorale et à l'inadéquation des compétences qui y sont développées. L'approche des compétences dans une telle perspective suppose une analyse des modes de production des connaissances comme maillon stratégique du développement social et économique (Carayannis et Campbell, 2017; Gibbons et al., 2012; Leydesdorff et Etzkowitz, 2000; Nowotny et al., 2001). Les orientations gouvernementales québécoises, quant à l'apport des universités, vont dans le même sens : " Dans le contexte d'une société basée sur le savoir et ouverte sur le monde, les universités québécoises doivent plus que jamais contribuer activement au développement social et économique " (Ministère de l'Éducation et de l'Enseignement supérieur, 2018, p. 9).

Toutefois, les travaux portant spécifiquement sur les processus en jeu dans le développement des compétences chez les doctorants et leur mise en œuvre demeurent assez rares dans le contexte canadien (Bangali et al., 2019; Desjardins, 2012; Desjardins et King, 2011; Melin et al., 2006). On pourrait alors se demander s'il y a réellement une inadéquation entre les acquis de la formation doctorale et les besoins des organisations, et quels seraient les processus en jeu. L'investigation de telles questions nécessite une approche théorique et méthodologique centrée sur l'articulation compréhensive de leurs dimensions subjective et contextuelle. En effet, ces questions évoquent des processus qui nous semblent bien plus complexes qu'une simple question de transfert de compétences. Elles renvoient à la complexité et à la dynamique des processus sous-jacents au développement des compétences qui comportent une certaine intentionnalité, comme souligné par Kahn et Rey (2016). Selon ces auteurs, la compétence serait déterminée par la visée qu'un individu a sur le monde. La prise en compte d'un tel principe semble incontournable dans l'analyse des difficultés d'insertion ou des questions relatives à l'inadéquation perçue des compétences des titulaires d'un doctorat par rapport aux besoins des organisations. Guidé par ces questionnements de fond, le travail d'analyse proposé dans cet article sera structuré autour des objectifs suivants : déterminer les perceptions que des titulaires d'un doctorat ont des compétences qu'ils ont pu développer grâce à leur formation; déterminer les perceptions que des responsables d'organisations ont des compétences de ces diplômés; effectuer une analyse comparative de ces perceptions à la lumière de certaines approches conceptuelles de la notion de compétence et en faire émerger des points de convergence.

\section{Cadre théorique}

La notion de compétence s'impose désormais au monde de l'éducation et des ressources humaines tout en ne cessant d'évoluer (Klieme et al., 2008; Le Boterf, 2015). Les changements sociaux et le développement d'une économie du savoir seraient à l'origine de cette évolution (Göransson et Brundenius, 2012; Lee et al., 2009; Melin et Janson, 2006; Usher, 2002). Ainsi, les programmes de formation sont en renouvellement continu et plusieurs universités ont développé des référentiels de compétences afin d'adapter leurs objectifs pédagogiques (Université Laval, 2015). Mais qu'en est-il des approches conceptuelles à l'origine de ces référentiels et quelles sont les compétences qui sont réellement développées dans le système éducatif, en l'occurrence dans les programmes de formation doctorale? La réponse à cette question demeure assez complexe et, comme le soulignent Kahn et Rey (2016), " La notion de compétence souffre d'une incertitude épistémologique » (p. 4).

En effet, nous pouvons remarquer dans la littérature un manque de consensus quant à la manière de définir cette notion (Rychen et Salganik, 2003) et une certaine ambiguïté par rapport à sa conceptualisation. Certains auteurs, comme Jonnaert (2011, 2014), considèrent la compétence comme un processus complexe d'adaptation à une situation, davantage que comme une accumulation de savoirs, savoir-faire ou savoir-être. Dans une telle perspective, une compétence est toujours le résultat d'un processus temporel complexe, dynamique, dialectique et constructif du traitement d'une situation. La compétence serait donc liée à la capacité de l'individu à mobiliser un ensemble de ressources personnelles, psychosociales et environnementales pour poser des actions qui s'avèrent efficaces dans une situation donnée. C'est la manière dont cette personne déploie ses savoirs (ses connaissances, sa compréhension de ladite situation), ses savoir-faire et ses savoir-être. Ce processus évoque également la capacité de l'individu à utiliser les ressources disponibles dans l'environnement, à faire face aux contraintes et obstacles inhérents à la situation, et à développer de nouveaux savoirs, savoir- 
faire et savoir-être afin de poser des actions efficaces et efficientes. La compétence aurait ainsi une dimension métacognitive qui renvoie au fait de comprendre les raisons sous-jacentes aux actions que l'on pose ainsi qu'à la capacité d'en apprendre de nouvelles (Jonnaert, 2011; Le Boterf, 2015). Une définition de la notion de compétence devrait par conséquent être assez englobante pour refléter son aspect multidimensionnel tout en demeurant assez précise pour être utile et pratique dans les milieux organisationnels et scolaires. Toutefois, une telle conception des compétences centrée sur la performance et l'efficacité (Dubar, 1996; Le Boterf, 2015) rencontre quelques critiques dans les travaux en éducation (Crahay, 2006; Dolz et Ollagnier, 2002). II en va de même pour les référentiels qui semblent construits sur la même logique.

Ainsi, en appui à ces critiques qui dénoncent une tentative de subordination de l'école au monde de l'entreprise, Pepin (2016) se base sur les travaux de Dewey pour développer une conception de la notion de compétence comme partie intégrante du processus de formation dans le système scolaire. De ce point de vue, l'expérience éducative, notamment l'apprentissage expérientiel, permet de développer des compétences. Dans cette perspective,

la compétence ne relève pas seulement de l'agir : la compétence est une action réfléchie, c'est-à-dire une action médiatisée par la réflexion. En ce sens, développer la compétence, ce savoir-agir en situation, c'est aussi développer une capacité d'analyse pour problématiser les situations indéterminées qui surviennent, juger des ressources qui seront nécessaires pour surmonter les problèmes rencontrés et les mettre en œuvre à bon escient pour rétablir le continuum expérientiel. (Pepin, 2016, p. 27)

Nous pouvons également citer dans le même ordre d'idée les travaux de Kahn et Rey (2016), qui, à partir d'une analyse critique des référentiels, proposent un modèle explicatif de la notion de compétence. Ce modèle nous semble particulièrement pertinent pour approcher cette question dans le cadre de la formation doctorale. Pour ces auteurs, la compétence ne se définit pas à partir de la performance dans l'exécution d'une tâche, mais plutôt par les processus sous-jacents à cette performance. Dans ce sens, une personne compétente serait « quelqu'un qui est capable de choisir lui-même et à bon escient les opérations à accomplir dans une situation non routinière appartenant à son domaine " (p. 7). Cette capacité d'effectuer des choix pertinents et de poser des actions adéquates dans de nouvelles situations est analysée par Perrenoud (1994) comme des schèmes de pensée et de perceptions, à distinguer du savoir-faire, qui a souvent une certaine connotation procédurale.

Une telle approche des compétences évoque la question de leur transférabilité (Starck, 2019). Dujardin (2013) souligne une grande variabilité dans la manière d'aborder cet aspect, présenté tantôt comme des compétences transférables, transversales ou génériques. L'idée de la transférabilité des compétences repose sur des principes de la psychologie cognitive selon lesquels " la démarche qui permet de répondre à une tâche ou une situation, une fois acquise par le sujet, pourra être appliquée à toutes les situations de même structure, même si celles-ci diffèrent par de nombreux caractères " (Kahn et Rey, 2016, p. 9). La définition proposée par Bélanger et al. (2004) va dans le même sens : " La compétence est transférable dans la mesure où elle peut être utilisée, transposée dans un contexte de travail autre que celui dans lequel elle a été acquise » (p. 29).

La problématique liée à la perception des compétences des titulaires d'un doctorat sera analysée à la lumière de ces différentes approches du concept de compétence.

\section{Méthodologie}

\section{Considérations éthiques}

Les données présentées dans l'article proviennent d'un projet financé en partie par les Fonds de recherche du Québec - Société et culture (FRQSC) ainsi que par l'Association des doyennes et des doyens des études supérieures au Québec (ADÉSAQ) et ses partenaires (projet réalisé en collaboration avec David Litalien, professeur à l'Université Laval). Ce projet a été soumis à l'approbation du Comité d'éthique de la recherche de l'Université Laval et la collecte des données a été effectuée en conformité avec les directives reçues de ce comité.

Cette recherche visait une description des compétences des titulaires d'un doctorat selon leurs propres perceptions, de même que selon les perceptions 
des responsables d'organisations. La méthodologie mise en œuvre repose sur une démarche mixte à devis séquentiel : une étude qualitative basée sur des entretiens semi-directifs, suivie de deux enquêtes par questionnaire. L'étude qualitative visait à recenser les perceptions que des titulaires d'un doctorat ont des compétences qu'ils ont pu développer grâce à leur formation, d'une part, et d'autre part celles que des responsables d'organisations ont des compétences de ces diplômés. Les deux enquêtes avaient pour objectif de comparer les compétences précédemment recensées et d'en faire émerger des points de convergence.

\section{Caractéristiques des participants à l'étude qualitative}

La collecte des données a été effectuée auprès de deux profils de participants : des titulaires d'un doctorat ayant au moins une année d'expérience professionnelle et des responsables d'organisations employant ou susceptibles d'employer ce profil de diplômés. Ces deux profils de participants ont été recrutés sur la base d'une technique d'échantillonnage intentionnelle (raisonnée). La taille de l'échantillon pour chaque population a été ajustée selon les principes de saturation empirique (Corbière et Larivière, 2014; Sandelowski, 1995).

Les critères appliqués pour le recrutement des participants titulaires d'un doctorat étaient les suivants : être diplômé d'un doctorat de 3e cycle (Ph. D.) ou d'un doctorat à visée professionnelle (D. Psy., DBA, D. Ed., etc.), à l'exclusion des doctorats de 1er cycle. Le lieu de résidence retenu, pour des raisons de faisabilité, a été circonscrit aux villes de Québec, Montréal et Sherbrooke. Les personnes devaient par ailleurs avoir au moins une année d'expérience professionnelle à la suite de l'obtention de leur diplôme, et cela dans le but d'examiner l'adéquation des compétences perçues comme ayant été acquises grâce à la formation doctorale aux exigences de la profession exercée.

Quatre-vingt-cinq (85) titulaires d'un doctorat ont participé aux entretiens. Soixante-dix-huit (78) participants étaient des salariés, quatre (4) des travailleurs autonomes et trois (3) des entrepreneurs. Ils provenaient de neuf (9) champs disciplinaires : arts, droit, lettres, sciences administratives, sciences appliquées, sciences de la santé, sciences de l'éducation, sciences humaines, sciences pures.
Quant aux responsables d'organisations, la définition des critères de recrutement visait deux profils : des employeurs réels et des employeurs potentiels, c'est-àdire des organisations susceptibles d'embaucher des diplômés de doctorat, mais qui n'en comptent pas parmi leurs employés. Le choix des participants a été effectué de manière à couvrir les domaines d'études ciblés par le projet et déjà mentionnés ci-dessus. Pour des raisons de faisabilité, cette étude a été circonscrite aux villes de Québec et Montréal.

Vingt et un (21) responsables d'organisations ont participé aux entretiens, dont 12 employeurs réels ( $E r)$ et 9 employeurs potentiels (Ep). Onze (11) participants appartenaient à des organisations privées, six (6) provenaient d'organisations associatives et quatre (4) d'organisations publiques.

\section{Instruments de mesure de l'étude qualitative}

La collecte des données a été effectuée par la méthode de l'entretien semi-directif individuel (Creswell et Poth, 2018; Flick et al., 2018). Deux guides d'entretien ont été conçus à cet effet : l'un à l'intention des titulaires d'un doctorat et l'autre, à celle des responsables d'organisations. Chaque entretien était d'une durée approximative de 30 à 90 minutes.

Les principales dimensions des entretiens réalisés auprès des titulaires d'un doctorat portaient sur leur parcours doctoral et la perception des compétences développées, ainsi que sur l'adéquation et la transférabilité de ces compétences dans l'exercice de leur profession. Concernant les responsables d'organisations, les entretiens visaient leurs perceptions des compétences développées grâce à la formation doctorale, la plus-value qu'ils percevaient dans ces compétences, ainsi que leur évaluation de la concordance ou des écarts éventuels entre les attentes d'organisations comme la leur et les compétences développées au doctorat.

\section{Participants aux enquêtes}

Ces enquêtes ont été réalisées auprès des titulaires d'un doctorat (Ph. D.) ayant obtenu leur diplôme dans une université québécoise entre 2005 et 2015 ainsi que des responsables d'organisations publiques, privées ou associatives qui emploient ou sont susceptibles d'em- 
ployer ce profil de diplômés. 2139 titulaires d'un doctorat provenant de champs disciplinaires similaires à ceux de l'étude qualitative ont rempli le questionnaire. Leur âge moyen était de 41,7 ans. La moitié des participants était composée d'hommes, soit 50,4\%, les femmes représentaient $48,5 \%$ des répondants, et les autres, 2,1\%. Concernant les responsables d'organisations, 215 ont rempli le questionnaire, dont 123 employeurs réels et $92 \mathrm{em}-$ ployeurs potentiels. Ces participants provenaient essentiellement de quatre types d'organisations : entreprise privée (46,1\%), fonction publique fédérale, provinciale ou municipale (22,4\%), entreprise parapublique (11,4 $\%$ ) et centre hospitalier (5\%).

\section{Instruments de mesure de l'étude quantitative}

À la suite des analyses qualitatives, une échelle de compétences a été conçue et mesurée dans le cadre de deux enquêtes. Cette échelle est composée de 45 items classés dans neuf catégories : résolution de problèmes, gestion et encadrement, recherche et traitement de l'information, communication et rédaction, collaboration et partenariat, éthique professionnelle, créativité et innovation, apprentissage et adaptabilité, qualités personnelles (Bangali et al., 2019). Ce regroupement a été inspiré par les travaux d'Évéquoz (2004), entre autres.

Ces enquêtes avaient pour objectif de déterminer le degré auquel les participants titulaires d'un doctorat pensent avoir acquis ou mobilisé chacune des compétences de l'échelle, et ensuite de comparer ces perceptions à celles des responsables d'organisations.

\section{Résultats et discussion}

La présentation des résultats est structurée autour des trois objectifs définis précédemment : déterminer les perceptions que les titulaires d'un doctorat ont des compétences qu'ils ont pu développer grâce à leur formation; déterminer les perceptions que les responsables d'organisations ont des compétences de ces diplômés; effectuer une analyse comparative de ces perceptions à la lumière des approches conceptuelles de la notion de compétence et en faire émerger les points de convergence.

L'analyse des deux premiers objectifs repose sur les résultats de l'étude qualitative. Celle du troisième objec- tif a été effectuée grâce à des analyses descriptives et à des comparaisons de moyennes standardisées de données issues des deux enquêtes.

\section{Résultats de l'étude qualitative}

Les corpus des entretiens ont été traités par la méthode Alceste (Marpsat, 2010; Reinert, 2003, 2009), qui est un logiciel d'analyse de données textuelles par catégorisation et classification hiérarchique avec une mesure des unités textuelles les plus significatives. Le but est d'étudier, à travers les lois de distribution du vocabulaire dans le corpus, des types de représentations qui émergent du discours analysé (Reinert, 2003). Ce traitement a été effectué sans aucune catégorisation préalable afin de préserver une certaine objectivité dans l'extraction du sens du contenu analysé. Des catégories ont été définies dans un deuxième temps en fonction des classes de discours les plus significatives (khi 2) déterminées par le logiciel.

\section{Perceptions que les titulaires d'un doctorat ont de leurs compétences}

II ressort de l'analyse du corpus des entretiens réalisés auprès des 85 participants titulaires de doctorat deux thèmes prédominants, ou classes. Ces thèmes sont caractérisés par des mots significatifs qui constituent des indicateurs du monde lexical à l'origine de la classification. Ces mots sont triés par ordre d'importance (valeur de khi 2). Le nombre total de mots recensés est de 3465 , dont 121 qui ont été analysés. 91 unités textuelles ont été classées, dont 70 pour la classe 1 (soit $76 \%$ ) et 21 pour la classe 2 (soit $24 \%$ ). L'indice de pertinence est estimé à $93 \%$. Ces données confirment la validité du traitement effectué sur le corpus.

L'analyse des mots significatifs de ces classes a permis au logiciel de recenser deux catégories dans le discours des participants : un univers de référence focalisé sur les compétences que nous qualifions de transversales (classe 1) et un deuxième plus centré sur un monde lexical que nous proposons de qualifier de compétences clés (classe 2). 


\section{Figure 1}

Rapport d'analyse Alceste du corpus des entretiens des titulaires de doctorat

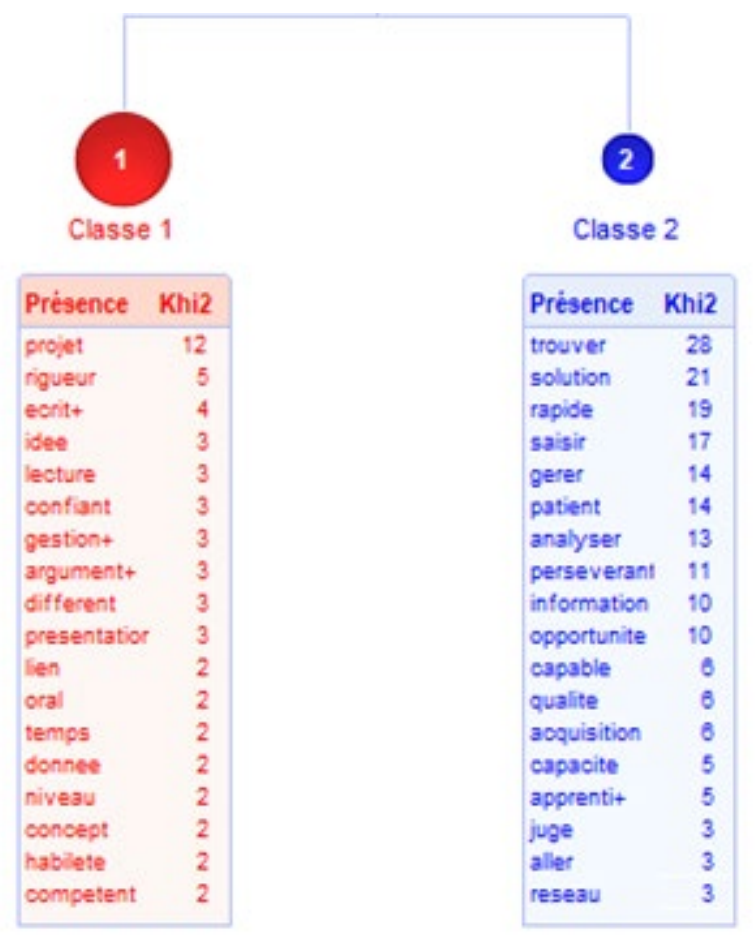

\section{Catégorie 1 : Les compétences transversales}

Cette première catégorie réfère à la classe 1 , qui est la plus spécifique. C'est la première qui s'est détachée dans l'arbre de classification. Son vocabulaire est le plus homogène. Elle représente $76 \%$ des unités textuelles classées et se caractérise par des mots (formes) tels que : projet, rigueur, écrits/écriture, idée, lecture, confiance, gestion, argumentation. Le thème qui émerge de l'analyse des unités textuelles de cette classe est en rapport avec les compétences qualifiées, dans les écrits, de transversales ou transférables, ou encore génériques, selon les auteurs (Bélanger et al., 2004; Dujardin, 2013; Kahn et Rey, 2016). À titre d'illustration, l'utilisation du mot projet est caractérisée par des énoncés qui sont en rapport avec le projet de recherche conduit dans le cadre de la thèse. Selon les perceptions de certains participants, la mise en œuvre de ce projet de recherche leur aurait permis de développer des compétences très utiles pour concevoir et mettre en œuvre des projets dans d'autres contextes. Un participant affirme par exemple que grâce aux acquis de sa formation doctorale, il observe dans son milieu de travail actuel qu'il est capable de " monter un projet de $A$ à $Z$ " (PhD5), et cela avec une certaine perspicacité qui le distingue des autres diplômés. Les commentaires d'un autre participant sur la conduite de projet vont dans le même sens. Ce dernier ajoute l'idée selon laquelle l'expérience du projet de recherche dans le cadre du doctorat lui aurait permis d'acquérir une grande capacité de " comprendre un problème, l'identifier et trouver les outils pour résoudre le problème " (PhD11). Il en va de même pour le fait de gérer et coordonner la mise en œuvre de projets de manière autonome. Selon certains participants, cette autonomie proviendrait du fait que dans certaines disciplines, le doctorant a été habitué à mener son projet de thèse seul. II a eu l'occasion de concevoir ce projet et en a réalisé toutes les étapes. Le mot rigueur réfère à la discipline, au fait de réaliser des activités dans le respect de certaines normes ou réglementations, mais également au souci de bien faire ainsi qu'au sens des responsabilités. À cela s'ajoutent des compétences rédactionnelles, l'argumentation des idées, l'intégration et l'analyse des données écrites: la lecture. L'extrait ci-dessous, tiré d'un des entretiens, constitue une illustration très pertinente qui résume bien 
l'univers lexical qui entoure la perception des acquis de la formation doctorale et de leur transférabilité :

Dans mon travail, ma formation doctorale m'aide à penser les mouvances actuelles et les défis qui se présentent en grand nombre sur le terrain. Elle m'a donné encore plus de rigueur et de capacité d'analyse et de synthèse des situations auxquelles nous faisons face comme organisation. (PhD8)

\section{Catégorie 2 : Les compétences clés}

L'analyse du vocabulaire particulier de cette catégorie permet de dégager un monde lexical centré sur les compétences qui pourraient être considérées comme étant spécifiquement liées au cœur de la formation doctorale. Cette catégorie représente $24 \%$ des unités textuelles classées. Les mots les plus significatifs de cette classe sont trouver, solution, rapide, saisir, gérer, patient, analyser, persévérant.

Les unités textuelles qui sont en lien avec le mot trouver évoquent la capacité à concevoir des stratégies pour trouver de l'information et l'organiser de façon intelligente afin de comprendre un sujet, réunir les éléments qui sont importants pour trouver une solution. À titre d'illustration, plusieurs unités textuelles montrent qu'une telle capacité repose sur une " compréhension fine des choses" (PhD20). Cela permettrait au titulaire d'un doctorat d'avoir une grande capacité d'analyse et de gestion d'une masse importante d'informations avec patience et persévérance, comme le met en exergue cette citation issue du discours d'un participant : " pour rédiger ma thèse, j'ai dû développer une capacité à comprendre rapidement ce qui est important sur un sujet et à réunir les éléments qui sont importants pour trouver une solution » (PhD15). Étant donné la complexité des recherches menées, les discours de cette catégorie laissent entendre que la formation doctorale permettrait au doctorant de développer une grande capacité à faire face aux problèmes complexes et à élaborer des stratégies pour les résoudre, comme l'illustre cet exemple : " de manière générale, la philosophie mène au développement d'une grande capacité à résoudre des problèmes et à s'adapter à différentes situations" (PhD30).

Ces résultats soulignent quelques points pertinents qui caractérisent les perceptions que des titulaires d'un doctorat peuvent avoir des compétences qu'ils con- sidèrent avoir développées grâce à leur formation. Ces éléments d'analyse évoquent certains aspects importants de la manière dont la notion de compétence est conceptualisée, notamment dans les travaux de Pepin (2016), comme partie intégrante du processus de formation. En effet, la manière dont ces participants perçoivent leurs compétences " ne relève pas seulement de l'agir, mais bien d'une action réfléchie. ... une capacité d'analyse pour problématiser les situations indéterminées qui surviennent, juger des ressources qui seront nécessaires pour surmonter les problèmes rencontrés et les mettre en œuvre à bon escient pour rétablir le continuum expérientiel » (p. 27). Aussi, dans la perspective d'auteurs comme Kahn et Rey (2016), qui proposent un modèle explicatif de la notion de compétence, nous pouvons noter dans les perceptions des participants que la compétence ne se limite pas à la performance dans l'exécution d'une tâche, mais inclut les processus sous-jacents à cette performance. Nous retrouvons cette idée dans l'évocation que certains participants font des processus d'analyse liés à la résolution de problème, au traitement de l'information, etc.

\section{Perceptions que les responsables d'organisations ont des compétences de ces diplômés}

L'analyse du corpus des entretiens réalisés auprès des 21 responsables d'organisations fait émerger également deux catégories de discours : l'une plus orientée sur des observations visant l'élaboration de stratégies pour faciliter l'employabilité des titulaires d'un doctorat (classe 1) et l'autre qui souligne la reconnaissance de certaines compétences, mais également des écarts entre les compétences des titulaires d'un doctorat et celles recherchées par les employeurs potentiels (classe 2).

Compte tenu des objectifs de cet article, qui vise à mieux comprendre les perceptions des participants sur les compétences des titulaires de doctorat, nous proposons de centrer notre analyse uniquement sur les énoncés de la deuxième catégorie (classe 2). Nous exclurons par conséquent la classe 1 , qui est moins pertinente pour notre analyse dans la mesure où elle évoque plutôt des conseils pour favoriser l'employabilité.

La catégorie 2 représente $71 \%$ des unités textuelles classées. Elle correspond à la classe 2 du dendrogramme (Figure 2). Les mots les plus significatifs de cette classe 


\section{Figure 2}

Rapport d'analyse Alceste du corpus des entretiens des responsables d'organisations

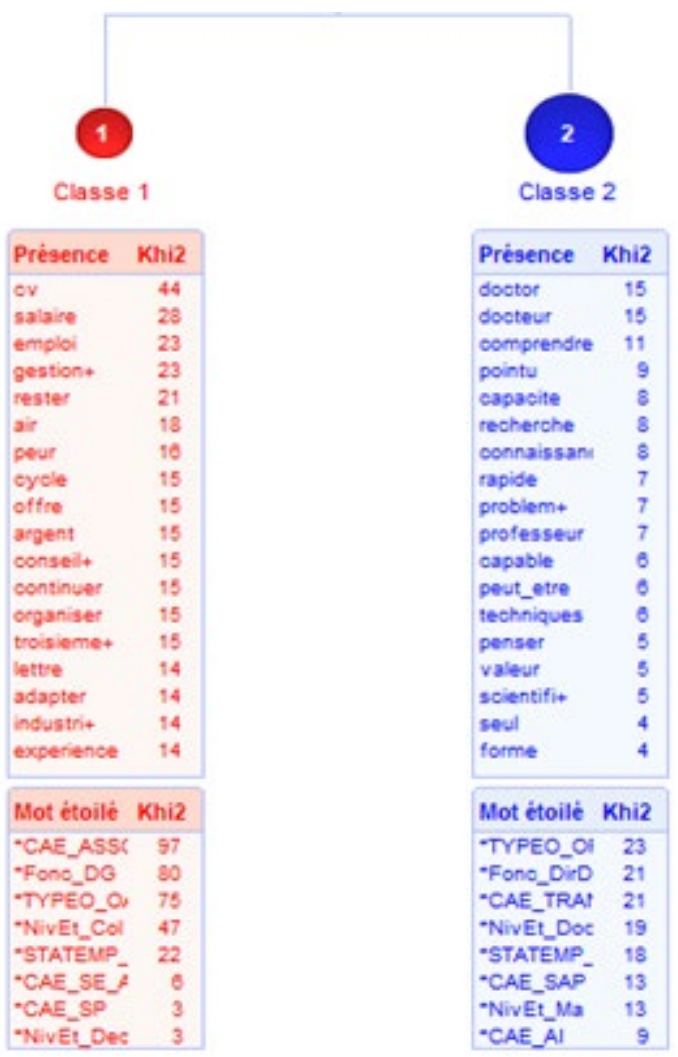

sont les suivants : doctorat, docteur, comprendre, pointu, capacité, recherche, connaissance.

L'analyse de l'univers lexical qui caractérise cette classe est orientée sur le fait que les titulaires d'un doctorat possèdent bien des compétences spécifiques, tout en soulignant certains aspects inadéquats. La capacité à comprendre ressort dans le discours des participants comme un point central des acquis de la formation doctorale qui semble très pertinent par rapport aux besoins de certaines organisations, comme le souligne ce participant :

Je vois la capacité d'analyser. La capacité de lire et de comprendre ce qu'on lit. Parce qu'on est souvent soumis à des plans, des devis ou des modèles. C'est énormément de lecture pour des hommes ou femmes techniques. Ils doivent comprendre exactement ce en quoi on s'engage. Donc je pense que c'est un point qui pourrait être très bien pour les doctorants, surtout si l'aspect pratique se développe. (Ep2)
Comme mentionné dans l'analyse précédente, cette perception des compétences des titulaires d'un doctorat ne semble pas se limiter à l'application efficiente de procédures. Elle évoque également les processus sous-jacents à l'exécution efficace de la tâche. Cette idée se manifeste dans plusieurs unités textuelles, dont celle présentée ci-dessous à titre d'illustration :

Des gens qui ont une vision globale, aussi. Ce sont des gens qui veulent comprendre le sens, qui ont une vision globale de l'organisation. Ils comprennent où est-ce qu'on s'en va, c'est quoi la valeur ajoutée de chacun des livrables dans le fond. (Er5)

L'idée principale qui émerge de l'analyse des discours associés aux mots les plus significatifs de cette classe semble marquée par une certaine ambivalence. II y a d'une part la reconnaissance que la formation doctorale permet le développement de connaissances pointues en recherche, une grande capacité à compren- 
dre les phénomènes en profondeur, mais aussi le constat d'une certaine inadéquation d'autre part. Une partie des discours rattachés au mot comprendre renvoie à cette réalité, comme le montre l'énoncé suivant:

Ce sont des gens qui ne sont pas forcément dans les délais, qui ne comprennent pas forcément l'urgence de produire quelque chose dans les délais. ... II faut agir rapidement, prendre des décisions, etc. Il y en a certains qui ont beaucoup de difficulté à prendre une décision s'ils n'ont pas tous les éléments en main. (Er8)

Ce constat d'une certaine déconnexion des exigences des organisations en dehors du milieu de la recherche et de l'enseignement semble résumer un aspect important des difficultés auxquelles certains titulaires d'un doctorat peuvent être confrontés dans leur transition. De plus, les analyses montrent que ces observations ne sont pas de simples préjugés, mais émanent du discours de participants qui ont des titulaires d'un doctorat comme employés au sein de leur organisation.

Par ailleurs, l'analyse du discours de certains responsables d'organisations met en exergue l'importance d'une prise en considération de ce que Kahn et Rey (2016) appellent l'intentionnalité. En effet, l'examen de ce qui pourrait être perçu comme une inadéquation des compétences des titulaires de doctorat, sans considérer les visées que ces derniers peuvent avoir sur le monde, serait une erreur. Comme le montrent Kahn et Rey (2016), la compétence implique également que l'individu ne réagisse pas à " des situations selon des mécanismes préétablis, mais comme porteur d'une intentionnalité qui consiste à poser des fins et qui lui permet de choisir de se conformer à telles normes ou bien à telles autres "(p. 16). Certains responsables d'organisations pointent parfaitement cet aspect en parlant des attentes et de la capacité du diplômé à les ajuster, comme illustré dans les trois énoncés suivants : "Ça dépend des attentes de la personne. Oui, elle a fait un doctorat. Est-ce qu'elle s'attend à utiliser l'ensemble de ces compétences-là dans un emploi? " (Er5); " Je pense que c'est compatible avec l'industrie, mais c'est une question de gestion des attentes " (Er10); « II faut que les détenteurs de doctorat réalisent et acceptent que les besoins de l'industrie sont différents de ce qui est vécu à l'université » (Er10).

Ces analyses nous amènent à déplacer l'axe central de ce qui est perçu comme une inadéquation vers l'intentionnalité. Ainsi, en paraphrasant Kahn et Rey (2016), on pourrait dire que certains diplômés d'un doctorat, jugés non compétents par des responsables d'organisations dans certains contextes, ne manqueraient peut-être pas forcément de ressources pour être performants, mais qu'en fonction d'une intention qui leur est propre, ils auraient probablement un autre regard sur les choses que celui qui est attendu par certaines organisations, regard sur le monde qu'ils auraient des raisons de privilégier.

\section{Résultats de l'étude quantitative}

L'analyse comparative des scores des responsables d'organisations par rapport à ceux des titulaires d'un doctorat aux items de l'échelle de mesure des compétences a permis de repérer des points de convergence, mais aussi de divergence de perceptions et d'en mesurer les écarts. Nous proposons de focaliser notre analyse sur les points de convergence.

\section{Analyse comparative des perceptions des titulaires d'un doctorat et de celles des responsables d'organisations}

L'analyse croisée des réponses des titulaires d'un doctorat et de celles des responsables d'organisations à l'échelle de mesure des compétences montre des points de convergence assez marqués pour 10 énoncés sur les 45. Le principe de cette convergence repose sur les énoncés dont la moyenne est assez élevée $(M \geq$ 4,40 ), aussi bien chez les responsables d'organisations publiques, privés et associative (ROPPA) que chez les titulaires d'un doctorat (PhD), avec une taille d'effet en dessous de $0,16(d<0,16)$, ce qui reflète une différence quasi nulle entre les perceptions. Ces énoncés font essentiellement référence à la capacité d'analyse et de synthèse, à la résolution de problèmes complexes, à la communication écrite comme orale, à l'esprit critique et à la créativité.

Ces résultats montrent que la formation doctorale pourrait constituer un atout pour le développement des compétences du futur, notamment celles qui sont difficiles à automatiser, " des compétences abstraites et complexes en matière de prise de décision, avec un fort accent sur la créativité, la réflexion critique et les aptitudes interpersonnelles et sociales » (Johal et Urban, 


\section{Tableau 1}

Points de convergence entre compétences acquises et compétences recherchées

\begin{tabular}{lccc} 
& PhD & ROPPA & Effect size \\
& $\boldsymbol{M}$ & $\boldsymbol{M}$ & $\boldsymbol{d}$ \\
\hline $\begin{array}{l}\text { 1. Analyser un phénomène complexe ou un contexte sous différents } \\
\text { angles. }\end{array}$ & 4,63 & 4,72 & $-0,13$ \\
$\begin{array}{l}\text { 2. Synthétiser et organiser efficacement un nombre important } \\
\text { d'informations. }\end{array}$ & 4,54 & 4,63 & $-0,12$ \\
3. Poser un regard critique sur les différentes ressources con- & 4,57 & 4,65 & $-0,10$ \\
$\quad$ sultées. & & & \\
4. Communiquer ses idées à l'oral de manière claire et structurée. & 4,45 & 4,48 & $-0,03$ \\
5. Communiquer ses idées à l'écrit de manière claire et structurée. & 4,58 & 4,57 & 0,01 \\
6. Analyser diverses situations avec un esprit critique. & 4,52 & 4,61 & $-0,12$ \\
7. Intégrer rapidement de nouvelles connaissances. & 4,40 & 4,45 & $-0,06$ \\
8. Travailler de manière rigoureuse. & 4,61 & 4,66 & $-0,07$ \\
9. Réaliser des tâches complexes de manière autonome. & 4,72 & 4,63 & 0,14 \\
10. Faire preuve de curiosité intellectuelle. & 4,66 & 4,59 & 0,10 \\
\hline
\end{tabular}

2020, p. 14). En effet, les énoncés qui ont les scores les plus élevés dans les points de convergence des perceptions des compétences des titulaires d'un doctorat sont en rapport avec certains aspects clés de ces compétences du futur, à savoir : la gestion de la complexité, la créativité, l'esprit critique. Les données du tableau 1 confirment ce constat :

- Analyser un phénomène complexe ou un contexte sous différents angles $(d=-0,13)$.

- Réaliser des tâches complexes de manière autonome $(d=-0,14)$.

- Intégrer rapidement de nouvelles connaissances $(d=-0,06)$.

- Faire preuve de curiosité intellectuelle $(d=$ $-0,10)$.

- Poser un regard critique sur les différentes ressources consultées $(d=-0,10)$.

- Analyser diverses situations avec un esprit critique $(d=-0,12)$.

Par ailleurs, il est possible de noter, dans les discours des participants titulaires de doctorat, l'apport des compétences développées au doctorat pour l'adaptation en emploi. Cependant, l'intentionnalité qui motive la mise en œuvre de ces compétences reste encore un défi pour certains diplômés. Comme le montrent les auteurs travaillant sur cette dimension des compétences (Kahn et Rey, 2016), celle-ci n'est pas forcément consciente, et cela pourrait être perçu par certains employeurs comme un manque d'adéquation.

\section{Limites}

Comme souligné dans le cadre théorique, la notion de compétence est très difficile à circonscrire et demeure marquée par un manque de consensus. De ce fait, les résultats présentés dans cet article reposent sur un choix délibérément orienté par une certaine vision de ce concept. Par ailleurs, nous sommes conscients que les spécificités disciplinaires peuvent avoir un impact sur les compétences développées, mais l'objectif de l'étude consistait à avoir une vision globale des perceptions que ces diplômés ont des compétences développées grâce à leur formation doctorale. Des études ultérieures 
plus fines seraient nécessaires pour se pencher sur les spécificités. Aussi, dans les analyses, il serait intéressant de démontrer la corrélation entre les compétences recensées et la formation doctorale. Des analyses plus approfondies avec tests statistiques pourraient être réalisées à cet effet. De plus, nous pourrions approfondir l'étude des conditions qui rendraient possible l'acquisition de ces compétences par une recherche longitudinale auprès de doctorants de divers champs disciplinaires.

\section{Conclusion}

Toutefois, malgré ces limites, les résultats présentés dans cet article montrent que la formation doctorale permet de développer des compétences très pertinentes face aux défis socioéconomiques et culturels du nouveau millénaire. Pour ce faire, il semble important d'en arriver à une meilleure démonstration de la plus-value du doctorat. Cela nous amène également à émettre des réserves concernant les données souvent diffusées dans les médias, qui tendent à insister sur le fait que les universités formeraient trop de doctorants, ou que les compétences de ces diplômés seraient inadéquates ou incompatibles. Les analyses et réflexions amorcées dans cet article nous poussent à remettre en question ces affirmations. La problématique à l'étude est beaucoup plus complexe qu'une simple question d'adéquation ou de transfert de compétences. Cela renvoie à la dynamique des processus de construction identitaire qui déterminent l'élaboration du projet personnel et professionnel chez les doctorants. L'analyse des processus sous-jacents au développement des compétences semble un aspect à approfondir pour mieux comprendre les fondements de l'intentionnalité qui oriente ce qu'un titulaire de doctorat a des raisons de valoriser dans l'exercice d'une profession.

\section{References}

Bangali, M., Litalien, D. et Guillemette, A. (2019). Étude panquébécoise sur les compétences des titulaires de doctorat [Rapport de recherche non publié]. Centre de recherche et d'intervention sur l'éducation et la vie au travail, Université Laval.

Bélanger, P., Legault, G., Beaupré, D., Voyer, B. et Trottier, M. (2004). La formation qualifiante et transférable en milieu de travail : recension des écrits, des pratiques et des enjeux [Rapport de recherche]. Université du Québec à Montréal. https://www.cpmt. gouv.qc.ca/publications/pdf/RECHERCHES T9 appels Bélanger2004 rapport.pdf

Braün, D. (2014, 7 mars). Faire de longues études pour mal gagner sa vie. Radio-Canada. https://ici.radio-canada.ca/nouvelle/656835/postdoctorants-canadiens-difficultes

Carayannis, E. G. et Campbell, D. F. J. (2017). Les systèmes d'innovation de la quadruple et de la quintuple hélice. Innovations, 54(3), 173-195.

Charbonneau, J. (2019, 11 novembre). Les défis d'être un travailleur surqualifié. Radio-Canada. https:// ici.radio-canada.ca/nouvelle/1384886/surqualification-doctorat-universite-emploi?depuisRecherche=true

Charrette, M. (2014, 11 mars). Peu d'emplois pour les diplômés du doctorat. Métro. https://journalmetro. com/carrieres/461460/peu-demplois-pour-lesdiplomes-du-doctorat/

Corbière, M. et Larivière, N. (2014). Méthodes qualitatives, quantitatives et mixtes dans la recherche en sciences humaines, sociales et de la santé. Presses de l'Université du Québec.

Crahay, M. (2006). Dangers, incertitudes et incomplétude de la logique de la compétence en éducation. Revue française de pédagogie, 154, 97-110.

Creswell, J. W. et Poth, C. N. (2018). Qualitative inquiry $\&$ research design: Choosing among five approaches. SAGE Publications.

Dalmont, A.-C. (2018, 29 octobre). Quels débouchés pour les titulaires d'un doctorat? 24h Montréal. https://www.24heures.ca/2018/10/29/quels-debouches-pour-les-titulaires-dun-doctorat

Desjardins, L. (2012). Profil et résultats sur le marché du travail des titulaires de doctorat des universités de I'Ontario (Catalogue no 81-595-M no 098). Statistique Canada. https://www150.statcan.gc.ca/n1/ pub/81-595-m/81-595-m2012098-fra.pdf

Desjardins, L. et King, D. (2011). Espérances et résultats sur le marché du travail des titulaires de doctorat des universités canadiennes (Catalogue no 81-595-M no 
089). Statistique Canada. https://www150.statcan. gc.ca/n1/pub/81-595-m/81-595-m2011089-fra.pdf

Didiano, T. J., Wilkinson, L., Turner, J., Franklin, M., Anderson, J. H., Bussmann, M., Reeve, D. et Audet, J. (2019, juin). I have a PhD! Now what? A Program to prepare engineering $P h D s$ and post-doctoral fellows for diverse career options [communication]. ASEE Annual Conference \& Exposition, Tampa, Florida. https://peer.asee.org/32910

Dolz, J. et Ollagnier, E. (2002). La notion de compétence : nécessité ou vogue éducative. Dans J. Dolz et E. Ollagnier (dir.), L'énigme de la compétence en éducation. Raisons éducatives (p. 7-24). De Boeck Université.

Dubar, C. (1996). La sociologie du travail face à la qualification et à la compétence. Sociologie du travail, 2 , 179-193.

Dujardin, J.-M. (2013). Compétences durables et transférables : clés pour l'employabilité. De Boeck.

Edge, J. et Munro, D. (2015). Inside and Outside the Academy: Valuing and Preparing PhDs for Careers. Conference Board du Canada. https://www. conferenceboard.ca/edu/research/valuing-preparing-phds-for-careers

Évéqu0z, G. (2004). Les compétences clés, pour accroître l'efficacité et l'employabilité de chacun. Éditions Liaisons.

Flick, U., Brinkmann, S., Kvale, S., Coffey, A., Barbour, R. S., Banks, M., Gibbs, G. et Rapley, T. (2018). The SAGE Qualitative Research Kit. SAGE Publications.

Gibbons, M., Limoges, C., Nowotny, H. et Schwartzman, S. (2012, 31 mai). The New Production of Knowledge: The Dynamics of Science and Research in Contemporary Societies. SAGE Publications. http:/l dx.doi.org/10.4135/9781446221853

Göransson, B. et Brundenius, C. (2012). L'université en transition : L'évolution de son rôle et des défis à relever. Springer-Verlag. https://doi. org/10.1007/978-1-4614-1236-6

Johal, S. et Urban, M. C. (2020, 20 janvier). Comprendre l'avenir des compétences: Tendances et réponses politiques dans le monde. Le Forum des politiques publiques. https://ppforum.ca/fr/publications/comprendre-lavenir-des-competences/

Jonnaert, P. (2011). Sur quels objets évaluer des compétences? Éducation et Formation, 296, 31-43.

Jonnaert, P. (2014). Évaluer des compétences? Oui, mais de quelles compétences s'agit-il? Dans $C$. Dierendonck, E. Loarer et B. Rey (dir.), L'évaluation des compétences en milieu scolaire et en milieu professionnel (p. 35-55). De Boeck Université.

Kahn, S. et Rey, B. (2016). La notion de compétence : une approche épistémologique. Éducation et francophonie, 44 (2), 4-18. https://doi. org/10.7202/1039019ar

Klieme, E., Hartig, J. et Rauch, D. (2008). The concept of competence in educational contexts. Dans J. Hartig, E. Klieme et D. Leutner (dir.), Assessment of competencies in educational contexts (p. 3-22). Hogrefe \& Huber Publishers.

Le Boterf, G. (2015). Construire les compétences individuelles et collectives : Agir et réussir avec compétence, les réponses à 100 questions (7e édition). Eyrolles.

Lee, A., Brennan, M. et Green, B. (2009). Re-imagining doctoral education: Professional Doctorates and beyond. Higher Education Research \& Development, 28(3), 275-287. https://doi. org/10.1080/07294360902839883

Leydesdorff, L. et Etzkowitz, H. (2000). Le « Mode 2 » et la globalisation des systèmes d'innovation " nationaux » : Le modèle à Triple hélice des relations entre université, industrie et gouvernement. Sociologie et sociétés, 32(1), 135-156. https://doi. org/10.7202/001434ar

Maldonado, V., Wiggers, R. et Arnold, C. (2013). So You Want to Earn a PhD? The Attraction, Realities, and Outcomes of Pursuing a Doctorate (Issue Paper No. 15). The Higher Education Quality Council of Ontario. https://heqco.ca/pub/issue-paper-no-15-soyou-want-to-earn-a-phd-the-attraction-realities-andoutcomes-of-pursuing-a-doctorate/

Marpsat, M. (2010). La méthode Alceste. Sociologie, 1(1). https://journals.openedition.org/sociologie/312 
McAlpine, L. et Austin, N. (2018). Humanities PhD Graduates: Desperately Seeking Careers? Canadian Journal of Higher Education, 48(2), 1-19. https://doi. org/10.7202/1057100ar

Melin, G. et Janson, K. (2006). What skills and knowledge should a PhD have? Changing preconditions for PhD education and post doc work. Portland Press. https://portlandpress.com/DocumentLibraryl Umbrella/Wenner\%20Gren/Vol\%2083/Chap11. Wenner Gren 83.pdf

Mendoza, P. (2007). Academic Capitalism and Doctoral Student Socialization: A Case Study. The Journal of Higher Education, 78, 71-96. https://doi.org/10.1353/ jhe.2007.0004

Ministère de l'Éducation et de l'Enseignement supérieur du Québec (2018). Politique québécoise de financement des universités. www.education.gouv.qc.ca

Nowotny, H., Scott, P. et Gibbons, M. (2001). Re-Thinking Science: Knowledge and the Public in an Age of Uncertainty. Polity Press.

Pepin, M. (2016). Le développement de compétences à l'école primaire au regard de la théorie de l'enquête de Dewey. Éducation et francophonie, 44(2), 19-39. https://doi.org/10.7202/1039020ar

Perrenoud, P. (1994). Compétences, habitus et savoirs professionnels. European Journal of Teacher Education, 17(1/2), 45-48. https://doi. org/10.1080/0261976940170108

Porter, S., Mol, L., Locher, J. et Johnson, M. (2017). UBC PhD Career Outcomes: Graduates from 2005-2013 UBC Vancouver Campus [Rapport de recherche]. University of British Columbia. https://outcomes. grad.ubc.ca/docs/UBC PhD Career Outcomes April2017.pdf

Reinert, M. (2003). The role of repetition in the representation of meaning and its statistical approach by the « ALCESTE » method. Sémiotique, 147, 389-420.

Reinert, M. (2009). Journée d'étude du 21 août 2009 sur la méthodologie "Alceste ». Arguments des interventions. Bulletin de méthodologie sociologique, 104, 39-46.
Reithmeier, R., O'Leary, L., Zhu, X., Dales, C., Abdulkarim, A., Aquil, A., Brouillard, L., Chang, S., Miller, S., Shi, W., Vu, N., Zou, C. (2019). The 10,000 PhDs project at the University of Toronto: Using employment outcome data to inform graduate education. PLoS ONE, 14(1). https://doi.org/10.1371/ journal.pone.0209898

Rychen, D. S. et Salganik, L. H. (2003). A holistic model of competence. Dans D.S. Rychen et L. H. Salganik (dir.), Key competencies for a successful life and well-functioning society (p. 41-62.). Hogrefe \& Huber Publishers.

Sandelowski, M. (1995). Focus on qualitative methods: Sample sizes in qualitative research. Research in Nursing \& Health, 18, 179-183.

Starck, S. (2019). Quelles relations entre conceptions " quotidienne " et scientifique des compétences transversales? Recherches en éducation, 37, 8-21. https://doi-org.acces.bibl.ulaval.ca/10.4000/ree.793

Tamburri, R. (2013, 6 février). Une réforme du doctorat s'impose. Affaires universitaires. https://www. affairesuniversitaires.ca/articles-de-fond/article/ une-reforme-du-doctorat-simposel

Université Laval (2015). Un guide de référence sur les compétences à développer à la maîtrise et au doctorat. https://www.fesp.ulaval.ca/sites/default/ files/documents/Publications avis rapports FESP/ rapport cfesp - guide - cetudes20150521 et 0604 - fesp20150721.pdf

Usher, R. (2002). A Diversity of Doctorates: Fitness for the knowledge economy? Higher Education Research \& Development, 21(2), 143-153. https://doi. org/10.1080/07294360220144060

\section{Contact Information}

\section{Marcelline Bangali}

marcelline.bangali@fse.ulaval.ca 


\title{
DOCTORAL GRADUATE SKILLS: A COMPARATIVE ANALYSIS OF PERCEPTIONS
}

\author{
MARCELLINE BANGALI \\ UNIVERSITÉ LAVAL
}

Note: This is an English translation of the original.

\begin{abstract}
A number of studies point to particular challenges that some $\mathrm{PhD}$ graduates face in the labour market outside of academia. One of the main reasons for these difficulties is said to be a lack of knowledge or inadequacy of what doctoral graduates have acquired in terms of skills sought by employers. However, apart from statistical data, there is little work that tells us about the perceptions that the various groups and individuals involved have of these skills. This article makes a contribution in this direction. It is based on the results of a sequential mixed methods study. The first phase consisted of a qualitative study using semi-structured interviews of 85 employed PhD graduates and 21 organizational leaders. The findings of this study, whose data were processed using the "Alceste method," were used to design a 45-item scale on the competencies of doctoral graduates. This scale was measured in two questionnaire surveys completed by 2,139 employed doctoral graduates and 215 organizational leaders. Descriptive analyses comparing standardized averages (Cohen's $\mathrm{d}$ ) highlight points of convergence that show that doctoral training could be an asset for the development of future skills, especially those that are difficult to automate: complexity management, creativity, critical thinking.
\end{abstract}

Keywords: PhD, transition, skills, future skills, intentionality, employability

\section{Résumé}

Plusieurs travaux soulignent des difficultés particulières auxquelles certains titulaires d'un doctorat sont confrontés sur le marché du travail en dehors du milieu universitaire. Une des principales raisons de ces difficultés serait la méconnaissance ou l'inadéquation des acquis de la formation doctorale en ce qui concerne les compétences recherchées par les organisations. Or, en dehors de données statistiques, peu de travaux nous renseignent sur les perceptions que les différents acteurs ont de ces compétences. Cet article apporte une contribution dans ce sens. Il est basé sur les résultats d'une recherche mixte à devis séquentiel. La première étape a consisté en une étude qualitative par entretiens semi-directifs réalisés auprès de 85 diplômés du doctorat en emploi et 21 responsables d'organisations. Les résultats de cette étude, dont les données ont été traitées par la méthode Alceste, ont servi à la conception d'une échelle de 45 items sur les compétences des titulaires d'un doctorat. Cette échelle a été mesurée lors de deux enquêtes par questionnaire auxquelles ont répondu 2139 diplômés du doctorat en emploi et 215 responsables d'organisations. Des analyses descriptives de comparaison de moyennes standardisées ( $d$ de Cohen) mettent en évidence des points de convergence qui montrent que la formation doctorale pourrait constituer un atout pour le développement des compétences du futur, notamment celles difficiles à automatiser : la gestion de la complexité, la créativité, l'esprit critique.

Mots-clés : doctorat, transition, compétences, compétences du futur, intentionnalité, employabilité

\section{Introduction}

Ideas advanced in the present article are attuned to recent media coverage of the challenges faced by doctoral graduates transitioning into the labour market (Braün, 2014; Charbonneau, 2019; Charrette, 2014; Dalmont, 2018; Tamburri, 2013). In Canada, there is a 
growing concern about issues related to graduate employability (Didiano et al., 2019; Edge \& Munro, 2015; McAlpine \& Austin, 2018; Porter et al., 2017; Reithmeier et al., 2019). For some authors (Maldonado et al., 2013; Mendoza, 2007), these issues could to a certain extent be traced back to the doctoral training itself and "mismatched" skills developed in it. Seen in this light, a competency-based approach implies examining knowledge production methods as a strategic link in social and economic development (Carayannis \& Campbell, 2017; Gibbons et al., 2012; Leydesdorff \& Etzkowitz, 2000; Nowotny et al., 2001). The Québec Government's policies on the role of universities pretty much follows the same line: "In a knowledge-based society that is open to the world, Québec universities must contribute more actively than ever before to social and economic development." (Ministère de l'Éducation et de l'Enseignement supérieur, 2018, p.9, free translation)

Yet studies on the development and application of skills among doctoral students that specifically focus on the processes involved remain scarce in Canada (Bangali et al., 2019; Desjardins, 2012; Desjardins \& King, 2011; Melin \& Janson, 2006). There is merit, then, in exploring whether there truly is a mismatch between acquired doctoral skills and employer needs as well as the processes involved. This type of inquiry calls for a theoretical approach and a methodology based on a comprehensive articulation of the subjective and contextual perspectives of these skills. This line of questioning, in fact, points to processes we believe are well beyond the mere transfer of skills. Processes driving skills development are both complex and dynamic and, as Kahn and Rey (2016) argue, involve a degree of intentionality. The latter submit that skills can be defined by an individual's outlook on the world-a principle that appears essential in examining the challenges of entering the labour market or any perceived mismatch between doctoral graduate skills and employer needs. Guided by these fundamental questions, the analysis proposed in the present article is structured around three key objectives: 1 ) identify the perceptions that doctoral graduates have of the skills they were able to develop through their training; 2) identify the perceptions that organizational leaders have of these skills; and 3) identify points of convergence in a comparative analysis of both, seen through different lenses of conceptual approaches to the notion of skill.

\section{Theoretical Framework}

The notion of skill is enjoying currency in education and human resources settings and is constantly evolving (Klieme et al., 2008; Le Boterf, 2015). Societal change and the development of a knowledge-based economy are behind this evolution (Göransson \& Brundenius, 2012; Lee et al., 2009; Melin \& Janson, 2006; Usher, 2002). As a result, programs of study are continually being updated and many universities have developed competency benchmarks in fine-tuning their training objectives (Université Laval, 2015). But what about the conceptual approach underpinning these benchmarks, and what skills are truly being developed in the education system andin our case-in doctoral training programs? The answer to this query is rather complicated, as stressed by Kahn and Rey (2016): "The notion of skill suffers from epistemological uncertainty " ( $p .4$, free translation).

We have noted, in fact, that there is indeed a lack of consensus in literature on how to define this notion (Rychen \& Salganik, 2003) and a degree of ambiguity regarding its conceptualization. Some scholars such as Jonnaert $(2011,2014)$ view skill as a complex process of adapting to a situation rather than simple acquisition of a body of knowledge, know-how and life skills. Seen this way, a skill is always the result of a temporal, complex, dynamic, dialectical and constructive process in handling a given situation. Skill is, then, the ability of the individual to capitalize on all available resources-personal, psychosocial and environmental-to undertake effective action in such a situation. It is how the individual harnesses his or her knowledge (expertise and understanding of the situation), know-how and life skills. This process also involves the individual's ability to draw on resources from the environment at hand, overcome inherent barriers and constraints and develop new knowledge, know-how and life skills in taking actions that prove to be efficient and effective. Skill also takes on a metacognitive dimension-in other words, understanding the underlying reasons for the actions we take and the ability to learn new ones (Jonnaert, 2011; Le Boterf, 2015). A definition of skill should consequently be encompassing enough to reflect its multidimensionality and still be accurate enough to be meaningful and applicable to educational and work settings. However, the idea of skills centred on performance and efficiency (Dubar, 1996; Le Boterf, 2015) is not without its critics in some education scholarship (Crahay, 2006; Dolz \& Ol- 
lagnier, 2002). The same applies to competency benchmarks that appear to have a similar underlying rationale baked into them. Thus, in echoing these critics decrying universities yielding to the corporate world, Pepin (2016) builds on the work of Dewey to develop a definition of skill as an integral part of the training process in the education system. In Pepin's interpretation, skills are developed from the educational experience, namely experiential learning:

Skill is not simply a matter of action, but also a calculated action, meaning action mediated through thought. Thus, developing a skill or know-how in a given situation also means developing an analytical ability to problematize situations that can occur, gauge the resources needed to solve the issues and effectively action them to re-establish the experiential continuum. (Pepin, 2016, p.27, free translation).

Similarly, through a critical analysis of competency benchmarks, Kahn and Rey (2016) propose an explanatory model of the notion of skill. This model appears to be particularly germane for us in examining the issue of doctoral training, as it sets out that skill cannot solely be defined by the performance of a task but rather by the processes driving this performance. In this respect, skilled individuals are "those able to effectively choose on their own the actions to be carried out in a non-routine situation in their field." ( $p .7$, free translation) This ability to make pertinent choices and appropriately follow through in new situations is discussed by Perrenoud (1994) as "patterns of thought and perception" distinguishable from know-how, which often has some procedural undertones.

This approach to skills raises the matter of their transferability (Starck, 2019). Dujardin (2013) emphasizes the considerable variability in this notion, with skills often defined as transferable, cross-disciplinary or generic. Transferable skills rest on principles of cognitive psychology according to which "the process which enables to respond to a task or situation, once acquired by the individual, can be applied to any situation of the same structure, even when many of the characteristics differ." (Kahn \& Rey, 2016, p.9, free translation) The definition proposed by Bélanger et al. (2004) follows in the same vein: "A skill is transferable when it can be used or translated into a work setting other than the one in which it was acquired." (p.29, free translation)

With all these different conceptual approaches to skill in mind, we will now examine issues related to the perception of doctoral graduate skills.

\section{Methodology}

\section{Ethical Considerations}

The data analyzed in the present article is drawn from a study project funded in part by the Fonds de recherche du Québec - Société et culture (FRQSC) and the Association des doyennes et des doyens des études supérieures au Québec (ADÉSAQ) and its partners. The study was conducted in partnership with Université Laval professor David Litalien, and was reviewed and approved by the Research Ethics Board of Universite Laval. Data were collected in strict adherence to the Board's guidelines.

The aim of the study was to provide a description of the skills of doctoral graduates based on their own perceptions and those of organizational leaders. The study used sequential mixed methods: a qualitative phase with semi-structured interviews, followed by two surveys employing questionnaires. The goal of the qualitative study was, on the one hand, to identify PhD graduates' perceptions of the skills they had developed through their doctoral training, and on the other, how organizational leaders saw the skills of these graduates. Both surveys were intended to compare skills previously surveyed and identify points of convergence.

\section{Qualitative Study: Participant Characteristics}

Data for the qualitative study were collected from two different cohorts: doctoral graduates with at least one year of work experience and organizational leaders employing or likely to employ these graduates. The cohorts were recruited using purposive (or judgemental) sampling. The sample size for each cohort was adjusted following the principle of empirical saturation (Corbière \& Larivière, 2014; Sandelowski, 1995).

Recruitment criteria for doctoral graduates (PhD) were as follows: being a doctoral graduate or having a professional doctorate (e.g. D. Psy., DBA, D. Ed, with the exception of medicine, pharmacy, veterinary). For the sake of feasibility, Québec City, Montréal and Sherbrooke were selected as places of residence. In order to gauge the alignment between job requirements and skills believed to have been developed through doctoral training, selected participants had to have had at least one year of work experience following graduation. 
Eighty-five (85) PhD graduates took part in the interviews. Seventy-eight (78) were employees, four (4) self-employed and three (3) entrepreneurs. They had graduated from nine (9) different disciplines: Arts, Law, Humanities, Business Administration, Health Sciences, Education and Social Sciences, and Pure and Applied Sciences.

As for the organizational leaders, recruitment criteria were narrowed to two profiles of employer: those who hire doctoral graduates $(A E)$ and those likely to hire them but without any currently on the payroll (PE). Selection of participants was done so as to match the fields of study targeted above. For the sake of feasibility, this survey was limited to Montréal and Québec City.

Twenty-one (21) organizational leaders participated in the interviews, including 12 actual employers (AE) and nine (9) potential employers (PE). Eleven (11) participants were from the private sector, six (6) from the non-profit sector, and four (4) from the public sector.

\section{Qualitative Study: Measurement Tools}

Data collection was carried out using semi-structured interviews, each lasting between 30 to 90 minutes (Creswell \& Poth, 2018; Flick et al., 2018). Two interview guides were designed: one for doctoral graduates and one for organizational leaders.

The main topics of the interviews with the former revolved around their doctoral training path and perceptions of the skills they developed, including the alignment and transferability of these skills in performing their jobs. As for the latter, the focus of the interviews was on their perceptions of skills developed through doctoral training, their thoughts on the added value of PhD skills and alignment or possible gaps between them and expectations in organizations like theirs.

\section{Quantitative Study: Survey Participant Characteristics}

Surveys were conducted using two cohorts: one comprised of doctoral graduates who had earned their degree from a university in Québec between 2005 and 2015 , and one comprised of organizational leaders from the public, private or not-for-profit sectors who either employed or were likely to employ these graduates. For the graduate survey, 2,139 PhD grads from disciplines similar to those in the qualitative study took part. The median age was 41.7 . Half of the respondents $(50.4 \%)$ identified as male, $48.5 \%$ as female and $2.1 \%$ as other. 215 respondents took part in the survey of organizational leaders, including 123 actual employers and 92 potential employers. Overall, these respondents represented four types of employer: private (46.1\%), public (federal, provincial or municipal bodies) (22.4\%), para-public (11.4\%), and healthcare (5\%).

\section{Quantitative Study: Measurement Tools}

Following data analysis, a competency scale was designed and validated in the two surveys. Largely inspired by the work of Évéquoz (2004) among others, the scale comprises 45 descriptors grouped into 9 categories: (1) Problem Solving, (2) Management and Guidance (3) Research and Processing Information, (4) Communication and Writing, (5) Teamwork and Partnership, (6) Ethics, (7) Creativity and Innovation, (8) Learning and Adaptability, (9) Personal Aptitudes (Bangali et al., 2019).

The purpose of the surveys was to determine the degree to which doctoral graduates thought they had developed and capitalized on each skill in the scale and then compare this perception with those of organizational leaders.

\section{Results and Discussion}

Our discussion of the key findings is structured around three set objectives: 1 ) identify the perceptions that doctoral graduates have of the skills they were able to develop through their training; 2 ) identify the perceptions that organizational leaders have of these skills; and 3) identify points of convergence in a comparative analysis of both, seen through different lenses of conceptual approaches to the notion of skill.

Our analysis of the first two objectives was based on the findings of the qualitative study. The third objective was subjected to descriptive statistical analyses and comparisons, then standardized to a common scale for the two sets of data.

\section{Qualitative Study Findings}

The corpus of interviews were first analyzed using the "Alceste method" (Marpsat, 2010; Reinert, 2003, 2009), 
where text analysis software of the same name generated, categorized and classified the data hierarchically, with a measurement of the most meaningful textual units. The goal was to study, using co-occurrences of words within the corpus, types of meaning that emerged from the discourse analysed (Reinert, 2003). To maintain objectivity in the extraction of content, the analysis was undertaken without any preset classification. Categories were then defined based on the most meaningful word classes (chi-square) identified by the program.

\section{Doctoral Graduates' Perceptions of their Skills}

The analysis of the corpus of 85 doctoral graduate interviews indicated two predominant themes or classes. These are characterized by meaningful words that are indicators of the lexical units forming the base of the classification. These words were sorted by descending hierarchical value (chi-square). In all, 3,465 words were identified, with 121 analysed. 91 textual units were then grouped into two classes, 70 (or $76 \%$ ) under Class 1, and 21 (or 24\%) under Class 2, with an estimated Relevance Index of $93 \%$, confirming the validity of the processing.

An analysis of meaningful words in these classes enabled us to identify two categories in the discourse of participants: one a set of references focused on competencies called cross-disciplinary (Category 1 ) and a second, more lexical-centred one that can be designated as key competencies (Category 2).

\section{Category 1: Cross-disciplinary Skills}

This category (Class 1 ), is the most specific, and the first to stand out in the classification tree. Its vocabulary is

\section{Figure 1}

Dendogram of Alceste analysis: Corpus of doctoral graduate interviews

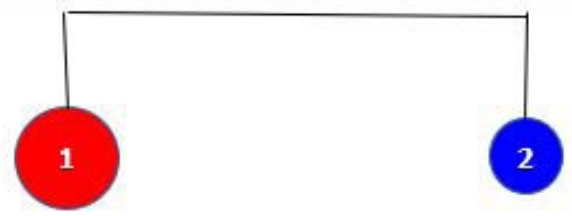

Classe 1

\begin{tabular}{|c|c|}
\hline Présence & Khi2 \\
\hline projet & 12 \\
\hline rigueur & 5 \\
\hline ecritt & 4 \\
\hline idée & 3 \\
\hline lecture & 3 \\
\hline confiant & 3 \\
\hline gestiont & 3 \\
\hline argument+ & 3 \\
\hline different & 3 \\
\hline presentation & 3 \\
\hline lien & 2 \\
\hline oral & 2 \\
\hline temps & 2 \\
\hline donnee & 2 \\
\hline niveau & 2 \\
\hline concept & 2 \\
\hline habilete & 2 \\
\hline competent & 2 \\
\hline
\end{tabular}

Classe 2

\begin{tabular}{|lr|}
\hline Présence & Khi2 \\
\hline trouver & 28 \\
solution & 21 \\
rapide & 19 \\
saisir & 17 \\
gerer & 14 \\
patient & 14 \\
analyser & 13 \\
perseverant & 11 \\
information & 10 \\
opportunite & 10 \\
capable & 6 \\
qualite & 6 \\
acquisition & 6 \\
capacite & 5 \\
apprentit & 5 \\
juge & 3 \\
aller & 3 \\
reseau & 3 \\
\hline
\end{tabular}


the most homogeneous, representing $76 \%$ of the categorized textual units, and is characterized by words or word forms such as: project, rigour, writing/text, idea, reading, confidence, management, and argumentation. The theme that emerges from analyzing the textual units in this class is related to those skills scholars define as cross-disciplinary, transferable or generic (Bélanger et al., 2004; Dujardin, 2013; Kahn \& Rey, 2016). For example, the use of the word project is accompanied by statements about research projects carried out as part of a thesis. Based on the perceptions of some respondents who carried out this type of project, it helped them develop very useful skills for planning and carrying out projects in other settings. One respondent noted, for example, that because of the skills developed through his doctoral thesis, in his current job he was able "to execute a project from start to finish" (PhD5), bringing an insight that set him apart from other graduates. Another respondent's comment on leading a project concurs, adding the idea that having had experience with a research project as part of his doctoral studies helped him to hone his ability to "understand the issue, identify it and find the tools to solve it." (PhD11) The same is true of independently managing and coordinating the rollout of a project. For some respondents, this independence comes from the fact that in some disciplines graduate students are used to leading a project, planning and executing every step on their own. The word rigour implies not only thorough discipline and carrying out activities in adherence with standards and regulations, but also diligence and a keen sense of responsibility. Added to this are writing skills, defending ideas, integrating and analyzing data: reading. Taken from one of the interviews, the quote below clearly exemplifies the lexical field surrounding perception of acquired doctoral skills and their transferability:

In my work, my doctoral training helps me think in terms of prevailing trends and the great many challenges we see in our field. It has given me even more discipline and the ability to analyze and synthesize situations which we are dealing with as an organization. (PhD8)

\section{Category 2: Key Skills}

Analyzing the vocabulary specific to Class 2 enabled us to identify a lexical field focused on skills considered to be at the core of doctoral training. This category represents $24 \%$ of the indexed textual units, with the most significant words being: find, solution, fast, understand, manage, patient, analyze and persevering.

Textual units related to the word find convey the ability to devise smart strategies for finding and organizing information in order to fully grasp a subject matter, and gathering all important elements needed for a solution. For example, several textual units show that this ability rests on "a keen understanding of things." (PhD20) This gives the doctoral graduate a greater ability to analyse and manage massive amounts of information with patience and perseverance, as illustrated in the words of this respondent: "To write my thesis, I had to develop an ability to quickly understand what was important for my topic and gather together all the important elements needed for a solution." (PhD15) Given the complex nature of research, the language in this category suggests that a doctoral training program could help a PhD student acquire greater skill in addressing complex issues and developing strategies for tackling them, as illustrated by this example: "Generally speaking, philosophy leads one to develop a great ability for problem solving and adaptability to different situations." (PhD30).

These findings underscore some of the relevant points exemplifying doctoral graduates' own perceptions of the skills they believe their training helped them develop. These insights also suggest some important aspects of the way the notion of skill is conceptualized - notably in the work of Pepin (2016) - as an integral part of the training process. In fact, these respondents perceived their skills "not simply a matter of action, but also a calculated action [...] an analytical ability to problematize situations that can occur, gauge the resources needed to solve the issues and effectively action them to re-establish the experiential continuum." (p.27, free translation). Following Kahn and Ray's (2016) explanatory model on the notion of skill, we noted that the perception of this notion among some of the participants is not limited to performance in executing a task, but also includes the processes driving this performance. This can be seen in references made by participants to analytical processes involved in problem solving and information processing, for example.

\section{Organizational Leaders' Perceptions of Doctoral Graduate Skills}

The analysis of the corpus of interviews conducted with 21 organizational leaders also produced two categories of discourse: the first centred on observations related 
to the employability strategies of doctoral graduates (Class 1) and the second on both recognition of doctoral graduate skills and gaps between these skills and those sought by potential employers (Class 2).

Given our objective to gain a better understanding of respondents' perspectives on PhD skills, we elected to exclude the first category (less relevant as it relates more to advice to increase employability) from our analysis and focus solely on the second, representing $71 \%$ of the indexed textual units and corresponding to Class 2 of the dendrogram in Figure 2. The most meaningful words in this class are: doctorate, $P h D$, understand, specialized, ability, research, and knowledge.

The analysis of the vocabulary emerging from this class highlights the fact that PhD graduates do have specific expertise while revealing some shortcomings. Based on the discourse of the respondents, the ability to understand stands out as the entire point of doctoral

\section{Figure 2}

Dendogram of Alceste analysis: Corpus of organizational leader interviews

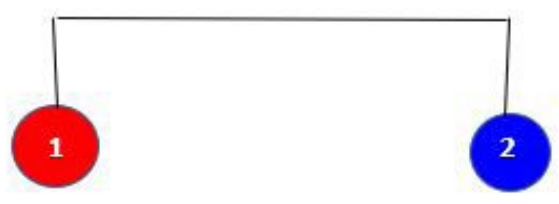

\begin{tabular}{|c|c|c|c|}
\hline \multicolumn{2}{|c|}{ Présence Khi2 } & Présence & Khi2 \\
\hline CV & 44 & doctor & 15 \\
\hline salaire & 28 & docteur & 15 \\
\hline emploî & 23 & comprendre & 11 \\
\hline gestion+ & 23 & pointu & 9 \\
\hline rester & 24 & capacite & 8 \\
\hline air & 18 & recherche & 8 \\
\hline peur & 16 & connaissance & 8 \\
\hline cycle & 15 & rapide & 7 \\
\hline offre & 15 & problem+ & 7 \\
\hline argent & 15 & professeur & 7 \\
\hline conseilt & 15 & capable & 6 \\
\hline continuer & 15 & peut_etre & 6 \\
\hline organiser & 15 & techniques & 6 \\
\hline troisiemet & 15 & penser & 5 \\
\hline lettre & 14 & valeur & 5 \\
\hline adapter & 14 & scientifit & 5 \\
\hline industrit & 14 & seul & 5 \\
\hline experience & 14 & forme & 4 \\
\hline
\end{tabular}

\begin{tabular}{|c|c|c|c|}
\hline Présence & Khi2 & Présence & Khi2 \\
\hline cv & 44 & doctor & 15 \\
\hline salaire & 28 & docteur & 15 \\
\hline emploì & 23 & comprendre & 11 \\
\hline gestion+ & 23 & pointu & 9 \\
\hline rester & 24 & capacite & 8 \\
\hline air & 18 & recherche & 8 \\
\hline peur & 16 & connaissance & 8 \\
\hline cycle & 15 & rapide & 7 \\
\hline offre & 15 & problem+ & 7 \\
\hline argent & 15 & professeur & 7 \\
\hline conseil+ & 15 & capable & 6 \\
\hline continuer & 15 & peut_etre & 6 \\
\hline organiser & 15 & techniques & 6 \\
\hline troisieme+ & 15 & penser & 5 \\
\hline lettre & 14 & valeur & 5 \\
\hline adapter & 14 & scientifit & 5 \\
\hline industrit & 14 & seul & 5 \\
\hline experience & 14 & forme & 4 \\
\hline
\end{tabular}

training, and appears to be very applicable to the needs of some employers, as pointed out by one of the respondents:

I see the ability to be analytical. The ability to read and understand what has been read. Because we often deal with plans, specifications or models. That's a lot of reading for technical men and women. You have to understand exactly what you're are getting into. So I think this is something doctoral students would be good at, especially if the practical side is developed. (PE2)

As noted in the discussion above, here too it appears that the perception of doctoral graduate skills is not limited to efficiently carrying out procedures. It also includes the underlying processes in effectively executing the task. This idea is found in many textual units, including in the following example: 
People who have a holistic vision, too. These are people who wish to understand the meaning, who have a holistic vision of work. They understand where we're headed and ultimately the added value of each of the deliverables (AE5).

The main point emerging from the analysis of the discourse related to the most meaningful words in this class appears to be marked by some ambivalence: There is a recognition on the one hand that doctoral training enables the development of specialized knowledge in research and a great ability to understand phenomena in depth, but also a certain misalignment on the other. A number of statements containing the word understand reflect this ambivalence, as shown in the following:

These are people who don't always work with deadlines, who don't necessarily understand the urgent need to produce something before one. [...] You need to act quickly, take decisions, etc. There are some who have trouble making decisions before they have all the information. (AE8)

This reservation speaks volumes to a disconnect between work demands outside the worlds of university research and academia, and captures an important aspect of the challenges faced by some $\mathrm{PhD}$ graduates as they transition into the job market. In addition, our findings show that these statements are not mere biases, but in fact stem from the discourse of respondents who count doctoral graduates among their employees.

Moreover, parsing the organizational leaders' discourse sharply puts into focus the need to consider Kahn and Ray's (2016) intentionality. Examining what might be construed as misalignment of a doctoral graduate's skills without also including the latter's outlook on the world would indeed be a mistake. As Kahn and Ray (ibid.) have shown, skill also means the individual does not merely react to "situations according to preestablished mechanisms, but as a bearer of an intentionality which consists in setting a purpose and which allows the bearer to choose whether to conform or not to one standard or another." (p.16, free translation). Some organizational leaders zero in on this aspect by referring to expectations and the ability of graduates to adjust to them, as illustrated in the following three statements: "It depends on personal expectations. Okay, they've got their PhD. Do they expect to use all these skills in a job?"
(AE5); "I think it's compatible with the industry but it's a matter of managing expectations" (AE10); "It's important that those with a PhD understand and accept that industry's needs are different than those they encountered in university." (AE10)

These analyses provide evidence to shift the focus away from the notion of a perceived mismatch towards intentionality. Thus, to paraphrase Kahn and Ray (ibid.), it could be argued that some doctoral graduates deemed by organizational leaders as not being skilled in a given setting is not because they lack the resources to perform well, but rather because of intentional objectives of their own, likely a vision of things different than those expected by some employers, an outlook on the world they have reasons to prioritize.

\section{Quantitative Study Findings}

A comparative analysis of doctoral graduate and organizational leader scores on the competency scale reveals both points of convergence and divergence in perceptions, and enabled us to measure the gaps. For purposes of this discussion we elected to focus on points of convergence.

\section{Points of Convergence Between Doctoral Graduates and Organizational Leaders}

The comparative analysis shows strong points of convergence in 10 of the 45 descriptors. The basis of this convergence is statements with very high averages $(M \geq$ 4.40) from both organizational leaders-be they public, private or not for profit (PPNL) - and doctoral graduates $(\mathrm{PhD})$, with an effect size of $0.16(d<0.16)$, reflecting almost no variance between perceptions. These statements refer mainly to analytical and synthesis skills, solving complex problems, as well as written and oral communication, critical thinking and creativity.

Our findings show that doctoral training can be an asset for future skills development, particularly those skills difficult to automate: "abstract, complex-decision-making skills with a strong focus on creativity, critical thinking and interpersonal social skills." (Johal \& Urban, 2020, p.13). 


\section{Table 1}

Points of convergence between acquired and desired skills

\begin{tabular}{lccc}
\hline & PhD & PPNL & Effect size \\
& $\boldsymbol{M}$ & $\boldsymbol{M}$ & $\boldsymbol{d}$ \\
\hline $\begin{array}{l}\text { 1. Analyze a complex phenomenon or context from different } \\
\text { approaches. }\end{array}$ & 4.63 & 4.72 & -0.13 \\
$\begin{array}{l}\text { 2. Efficiently synthesize and organize large amounts of } \\
\text { information. }\end{array}$ & 4.54 & 4.63 & -0.12 \\
3. Think critically about different resources used. & & & \\
4. Communicate ideas orally in a clear and organized manner. & 4.45 & 4.65 & -0.10 \\
5. Communicate ideas in writing in a clear and organized & 4.58 & 4.57 & -0.03 \\
manner. & & & 0.01 \\
6. Critically analyze different situations. & 4.52 & 4.61 & -0.12 \\
7. Quickly absorb new knowledge. & 4.40 & 4.45 & -0.06 \\
8. Work in a rigorous manner. & 4.61 & 4.66 & -0.07 \\
9. Independently accomplish complex tasks. & 4.72 & 4.63 & 0.14 \\
10. Show intellectual curiosity. & 4.66 & 4.59 & 0.10 \\
\hline
\end{tabular}

Statements with the highest points of convergence of perceptions of doctoral graduate skills tend to be those related to key elements of future skills, that is: complexity management, creativity, critical thinking. Data found in Table 1 confirm this finding:

Analyze a complex phenomenon or context from different approaches $(d=-0.13)$.

Independently accomplish complex tasks $(d=-0.14)$.

Quickly absorb new knowledge $(d=-0.06)$.

Show intellectual curiosity $(d=-0.10)$.

Think critically about different resources used $(d=$ $-0.10)$.

Critically analyze different situations $(d=-0.12)$.

In addition, the applicability on the job of expertise acquired in doctoral training can be gleaned from some of the discourse of PhD graduates. However, the intentionality which drives applying these skills remains a challenge for some graduates. As shown by scholars who have studied this aspect of skills (Kahn and Rey, 2016), this intentionality may not be conscious, and this in some cases can be perceived by certain employers as a "skills mismatch."

\section{Limitations}

As discussed in our theoretical framework, the notion of skill is rather difficult to pin down, and one marked by a lack of consensus. Hence the findings of the present article are the result of deliberate choices guided by a specific interpretation of this notion. We also recognize that some discipline specificities may have an impact on skills development. Nevertheless, the objective of the study was to provide a comprehensive picture of the perspectives doctoral graduates have of the skills they developed through their doctoral training; more in-depth studies are needed to further examine the issue in detail. The correlation between identified skills and doctoral training would also merit further study, and extensive research with statistical tests would certainly benefit scholarship in this area. Lastly, there is room to longitudinally study the optimal conditions for students developing doctoral skills different fields of study. 


\section{Conclusion}

Despite our stated limitations, the findings in the present article show that doctoral training allows the development of skills particularly relevant to tackling the social, economic and cultural challenges of the new millennium. It therefore appears imperative to better validate the added value of a PhD degree. We would, however, add a word of caution regarding data frequently quoted in the media that tend to focus on the fact that universities are training far too many PhDs, or that the skills of these graduates are either inadequate or mismatched. Indeed, our findings vigorously challenge these assertions. The issue in question is far more complex than a mere matter of aligning or transferring skills. It can be traced back to the dynamic processes of forging a personal identity in doctoral students that determines how personal and career plans are developed. To gain a fuller understanding of the foundation of intentionality underpinning what doctoral graduates value in the practice of their profession, a comprehensive examination of the underlying processes in skills development is needed.

\section{Contact Information}

Marcelline Bangali

marcelline.bangali@fse.ulaval.ca 